\title{
Comportamiento organizacional y su importancia en la auditoría pública
}

\section{Organizational behavior and its importance in public auditing}

\section{Comportamento organizacional e sua importância na auditoria pública}

\author{
Walter M. Mendoza-Zamora ${ }^{\mathrm{I}}$ \\ walter.mendoza@unesum.edu.ec \\ Jessenia H. Morán-Chilán II \\ jessenia.moran@unesum.edu.ec \\ Vanessa G. Fernández-Álava III \\ vanessa.fernandez@uleam.edu.ec \\ Ana del Rocío Lino-Calle IV \\ anali1979@hotmail.es
}

Correspondencia: walter.mendoza@unesum.edu.ec

I. Docente de la Universidad Estatal del Sur de Manabí, con más de 8 años de experiencia interuniversitaria, Gestor Financiero, Ingeniero Comercial, Master en Gestión Empresarial, graduado en la Universidad Técnica Particular de Loja (UTPL); Jipijapa; Ecuador.

II. Docente Titular de la Universidad Estatal del Sur de Manabí, con más 12 años de experiencia universitaria, Economista y Master en Economía Agraria, Coordinadora del Departamento de Cultura de la UNESUM; Jipijapa; Ecuador.

III. Ingeniera en Comercio Exterior y Negocios Internacionales, Magister en Administración de Empresas con Mención en Marketing por la Universidad de Guayaquil, docente por más de dos años en las carreras de Comercio Exterior y Economía de la Facultad Ciencias Económicas de la Universidad Laica Eloy Alfaro de Manabí; Manta; Ecuador.

IV. Licenciada en Administración de Empresas Agropecuarias, con experiencia en docencia universitaria y se desempeña actualmente como docente de la Universidad Estatal del Sur de Manabí; Jipijapa; Ecuador. 


\section{Resumen}

Este trabajo sintetiza el conocimiento del comportamiento organizacional y la importancia que esto genera al momento de una auditoria pública, con el fin de preparar a las empresas para las evaluaciones que las auditorias ameritan en el departamento administrativo, considerando que el área financiera es la más vulnerable en estos temas. A través de las auditorias se puede identificar los errores cometidos en las organizaciones y se puede enmendar a tiempo cualquier falla en la ejecución de la estrategia, permitiendo tomar medidas necesarias que permitan retomar el rumbo correcto en la empresa. El trabajo hace mención a la necesidad de realizar auditorias cuando existen evidencias de gastos excesivos, incumplimiento de proyectos, quejas de los clientes, alta rotación de recursos humanos y cuando los resultados se alejan de los que la empresa espera. Es conveniente que el auditor conozca y aplique, siempre que sea posible los métodos de medición cuantitativa y cualitativa, ya que, mucho de los problemas administrativos que se han presentado con el avance del tiempo, han generando nuevas dimensiones en el pensamiento administrativo y han considerando una de las principales y más importantes evaluaciones a la auditoria pública, presentado como un examen detallado de la administración por parte de un organismo social y realizado por un profesional llamado auditor. Tomando en consideración que los procesos administrativos van ligados al comportamiento organizacional que existe entre los miembros de una empresa y que son ellos los realmente responsables de la eficiencia y eficacia del cumplimiento de los objetivos, se tiene, que la auditoría pública es una actividad empresarial sumamente necesaria es estos tiempos.

Palabras claves: Administración; Control Interno; Estado; Evaluación; Supervisión.

\section{Abstract}

This work summarizes the knowledge of organizational behavior and the importance that this generates at the time of a public hearing, in order to prepare companies for evaluations that audiences in the administrative department, the financial area is the most vulnerable in these themes Through audits you can identify the mistakes made in the organizations and you can amend at a time any failure in the execution of the strategy, the necessary measures to return to the right direction in the company. The work refers to the need to conduct audits when there is evidence of excessive spending, the fulfillment of projects, the high turnover of human resources and the result of the company expects. It is convenient for the auditor to know and apply, whenever possible, the methods of quantitative and qualitative measurement, which, much as the problems that have arisen 
with the advance of time, have generated new dimensions in administrative thinking and have been A public society guide, a report of a detailed examination of the administration, a part of a social organization and a professional called an auditor. Take into account that the administrative processes are linked to the organizational behavior that exists between the members of a company and that are responsible for the efficiency and compliance with the objectives, one has, that the public audit is a highly necessary business activity is these time.

Keys words: Administration; Internal control; State; Evaluation; Supervision.

\section{Resumo.}

Este trabalho resume o conhecimento do comportamento organizacional e a importância que isso gera no momento de uma audiência pública, a fim de preparar as empresas para avaliações que audiências no departamento administrativo, a área financeira é a mais vulnerável nesses temas Através de auditorias você pode identificar os erros cometidos nas organizações e você pode corrigir de uma só vez qualquer falha na execução da estratégia, as medidas necessárias para voltar à direção certa na empresa. $\mathrm{O}$ trabalho refere-se à necessidade de realizar auditorias quando há indícios de gastos excessivos, o cumprimento de projetos, a alta rotatividade de recursos humanos e o resultado esperado pela empresa. É conveniente que o auditor conheça e aplique, sempre que possível, os métodos de mensuração quantitativa e qualitativa, que, assim como os problemas que surgiram com o avanço do tempo, geraram novas dimensões no pensamento administrativo e têm sido uma sociedade pública. guia, um relatório de um exame detalhado da administração, uma parte de uma organização social e um profissional chamado auditor. Levar em conta que os processos administrativos estão ligados ao comportamento organizacional que existe entre os membros de uma empresa e que são responsáveis pela eficiência e conformidade com os objetivos, tem-se, que a auditoria pública é uma atividade empresarial altamente necessária é nestes momentos.

Palavras chaves: Administração; Controle interno; Estado; Avaliação; Supervisão. 


\section{Introducción.}

Para hablar de comportamiento organizacional se debe tomar en consideración que la administración de empresas es un proceso de funciones básicas diferentes, como planificación, organización, dirección, ejecución y control, que permite enfrentar los retos que se desempeñan en una empresa.

Los autores (Gil, Ruiz, \& Ruiz, 1997) expresan que:

En la teoría administrativa, el concepto del comportamiento ha sido heredado de la economía y se considera como un principio rector, donde la evaluación del desempeño organizacional es importante pues permite establecer en qué grado se han alcanzado los objetivos, que casi siempre se identifican con los de la dirección, además se valora la capacidad y lo pertinente a la práctica administrativa. Sin embargo al llevar a cabo una evaluación simplemente a partir de los criterios de eficiencia clásico, se reduce el alcance y se sectoriza la concepción de la empresa, así como la potencialidad de la acción participativa humana, pues la evaluación se reduce a ser un instrumento de control coercitivo de la dirección para el resto de los integrantes de la organización y solo mide los fines que para aquélla son relevantes. Por tanto se hace necesario una recuperación crítica de perspectivas y técnicas que permiten una evaluación integral que involucre los distintos procesos y propósitos que están presentes en las organizaciones.

Sin embargo, el autor (Reyes A. 2005) en su estudio dice que:

El comportamiento organizacional es un campo de estudio en el que se investiga el impacto que individuos, grupos y estructuras tienen en la conducta dentro de las organizaciones con la finalidad de aplicar estos conocimientos a la mejora de la eficacia de tales organizaciones, 
se puede enunciar que es una disciplina científica a cuya base de conocimientos se agrega constantemente una gran cantidad de investigaciones y desarrollos conceptuales. Pero también es una ciencia aplicada, ya que, la información sobre prácticas efectivas en una organización puede extenderse a muchas otras y dejar así el departamentalismo.

De acuerdo a lo manifestado por los autores, se puede suponer que el comportamiento organizacional es como un campo de estudio de especialidad delimitada con un conjunto común de conocimientos que estudia tres determinantes del comportamiento de las organizaciones: individuos, grupos y estructura, y que su principal objetivo es estar al pendiente de las situaciones que atañen al empleo.

Los autores Sánchez y Bustamante (2008) comentan que:

El comportamiento organizacional se relaciona con la auditoría pública, ya que, esta consiste en la verificación, realizada con posterioridad y efectuada de forma sistemática, de la actividad económico-financiera del sector público, mediante la aplicación de los procedimientos de revisión selectivos contenidos en las normas técnicas de auditoría.

Por tal motivo la auditoría pública y el comportamiento organizacional son piezas muy importantes, ya que, son una actividades independientes de apoyo a la función directiva, enfocada al examen objetivo, sistemático y evaluatorio de las operaciones financieras y administrativas realizadas de los sistemas y procedimientos implantados; de la estructura orgánica en operación y de los objetivos, programas y metas alcanzados por las Dependencias, Órganos Desconcentrados y Entidades de la Administración Pública, así como de la Procuraduría General con el propósito de determinar el grado de economía, eficacia, eficiencia, efectividad, imparcialidad, honestidad y apego a la normatividad con que se han administrado los recursos públicos que les fueron suministrados, tal 
como la calidad y calidez con que prestan sus servicios a la ciudadanía, sin embargo, es un campo que aún no ha sido mayormente profundizado, por consiguiente, no son muchas las publicaciones que se encuentran y que hagan alusión al tema; por tal razón, la presentación de este artículo se realiza con el objetivo de dar a conocer algunos de los aspectos básicos, primordiales y que son importantes tanto para las organizaciones que llevan a cabo la evaluación del desempeño como también para los auditores que desarrollan su trabajo de revisión en estas áreas.

En este caso concreto, se tratará sobre el comportamiento organizacional y su importancia en la auditoría pública, que estará detallado a lo largo de este apartado.

\section{Desarrollo.}

\section{Materiales y métodos.}

La investigación está enfocada en el estudio del comportamiento organizacional y su importancia en la auditoría pública teniendo como misión ayudar a la dirección a lograr una administración eficaz para el desempeño en todas las áreas. Los factores de evaluación abarcan desde el panorama económico en que actúa una empresa, lo apropiado de la estructura organizativa, la observación de las políticas y procedimientos, la exactitud y confiabilidad de los controles, los métodos protectores adecuados, las causas de las variaciones, hasta la adecuada utilización de personal, equipo, materiales y los sistemas de funcionamientos satisfactorios, considerando todos los factores hasta conocer con profundidad los elementos de que está compuesto para verificar la viabilidad de los diversos procesos científicos, tomando en cuenta en que una de los principales auxiliares en que se basa la auditoria pública es la investigación científica, por lo cual el auditor público se apoya en la lógica siguiendo los pasos del razonamiento "inductivo y deductivo" que aconseja la decisión a tomar. 
El trabajo está basado en una revisión bibliográfica que conduce a un análisis crítico documental y que justifica, no solo la necesidad de auditar procesos sea de índole público o privado, sino también, que muestre que la teoría del comportamiento organizacional es la vía para garantizar la pulcritud de los procesos administrativos. La revisión se ha centrado en textos, documentos y artículos científicos publicados disponibles en la web, considerando que aquella herencia de la globalización nos permite acceder a mayor y mejor información a través de las herramientas tecnológicas. El motor de búsqueda ha sido herramientas académicas de la web que direccionan específicamente a archivos con validez y reconocimiento científico, descartando toda información no confirmada o sin las respectivas referencias bibliográficas.

\section{Definiciones de Comportamiento Organizacional}

“El Comportamiento Organizacional retrata la continua interacción y la influencia recíproca entre las personas y las organizaciones, es una disciplina académica que surgió como un conjunto interdisciplinario de conocimientos para estudiar el comportamiento humano en las organizaciones" (Chiavenato, 2009).

Los autores (Gibson, Ivancevich, Donnelly, \&Konopaske, 2007) lo definen como:

Campo de estudio que se sustenta en la teoría, métodos y principios de diversas disciplinas para aprender acerca de las percepciones, valores, capacidades de aprendizaje de los individuos mientras trabajan en grupos y dentro de la organización y para analizar el efecto del ambiente de la organización y sus recursos humanos, misiones, objetivos y estrategias. 
"Estudio de individuos y grupos en el contexto de una organización y el estudio de los procesos y prácticas internas que influyen en la efectividad de los individuos, los equipos y la organización" (Hellriegel\&Slocum, 2009).

También se puede definir qué "Es un campo de estudio que investiga el impacto de los individuos, grupos y estructuras sobre el comportamiento dentro de las organizaciones, con el propósito de aplicar los conocimientos adquiridos en la mejora de la eficacia de una organización” (Robbins, 2004).

De acuerdo a las definiciones presentadas, se puede observar las semejanzas entre estas al explicar que el comportamiento organizacional es la interacción, relación entre personas, grupos individuos y la organización, igualmente todos los autores de una u otra manera indican que es un campo de estudio, aplicación de conocimiento, disciplina, entre otros, utilizada para aumentar la confianza de la organización o mejorarla.

Ultimando en base a dichas definiciones y a criterio personal se tiene que, el objeto práctico en esta investigación, busca definir el Comportamiento Organizacional como el estudio de lo que las personas hacen en una organización, que distingue a las organizaciones unas de otras y que el objetivo principal es aplicar tal conocimiento para mejorar la organización. Por consiguiente se debe tener en cuenta que cada persona es un fenómeno multidimensional sujeto a las influencias de diversas variables, tanto externas como internas y que de estas depende el comportamiento humano.

\section{La cultura organizacional en las instituciones públicas}

La cultura organizacional según (Alles, 2008) quien en su obra expresa, "comportamiento organizacional es definida como una colección de valores esenciales compartidos, los cuales 
proveen mensajes explícitos e implícitos de los comportamientos preferidos en la institución; pero este difiere de ser el único concepto aceptado hoy día por los investigadores académicos globales”

Sin embargo, la abstracción sobre la cultura organizacional se inició en el anonimato absoluto, hace más de cuarenta años, por investigadores tales como Elliot Jaques, ErvingGoffman, Chester Barnard y Paul Selznik, quienes estudiaron aspectos como los valores y el estilo de dirección, no obtante, (Zapata, Murillo, \& Martínez, 2007) afirman que: el tema se encuentra en primer plano hoy en día, en razón de su relación con los problemas sociales, culturales, organizacionales e individuales, lo que ha permitido que el concepto adquiera mayor autonomía y sea estudiado con seriedad.

A pesar de la afirmación de los autores anteriores en que todo inicia en el anonimato y sin tener una fecha exacta,

Se puede asegurar algo sobre la perspectiva cultural de las organizaciones, y es su firme afianzamiento en los años setenta (70), cuando el concepto de empresa obtiene mayor complejidad. Así de una concepción de empresa basada estrictamente en criterios económicos, se ve, en los setenta a la empresa como una entidad social, por lo cual se le otorgan nuevas responsabilidades hacia su personal y hacia su entorno, y a la vez se hace más claro que la cotidianidad de las empresas tiene la complejidad propia de los fenómenos sociales (Thevenet, 2010).

En una institución intervienen numerosos factores que no pueden asumir ni los mejores modelos de gestión, un presidente exige a sus gerentes un gran rigor en la elaboración de un plan institucional, para después aconsejarles que lo guarden en un cajón; los directores se complacen en resumir en dos o tres slogans la razón de su éxito; décadas de auge empresarial e institucional se pretenden resumir 
en unos cuantos preceptos; estas realidades parecen apartarse de las complejas prácticas de gestión que esas mismas instituciones siguen utilizando.

Los autores (Zapata, Murillo, \& Martínez, 2007), opinan que:

El posicionamiento de la perspectiva de la cultura en las organizaciones también está íntimamente ligado a la transformación japonesa, la cual desde las cenizas de la segunda guerra mundial, construyó un poderoso imperio industrial a finales de los setenta y principio de los ochenta, irrumpió con una gran fortaleza y dominó en los mercados internacionales, a la vez que internamente obtuvo altas tasas de desarrollo y bajas tasas de desempleo; lo cual se explica según los teóricos de las organizaciones por una fuerte relación entre cultura y dirección organizacional. Dicha trasformación japonesa le demostró, en los años ochenta, al sorprendido occidente a través de diversos autores como Peters y Waterman, que desarrollar trabajos sobre temas de cultura organizacional es tan justificado como hablar de estructura organizacional, evidenciando a su vez, que los paradigmas administrativos tradicionales resultaron insuficientes para la comprensión de las organizaciones.

Hoy en día, el concepto de cultura organizacional es nuevo en cuanto su aplicación en la administración pública, es una nueva óptica, la cual le permite a la gerencia comprender y mejorar las instituciones. La cultura de una organización es un factor clave del éxito, por su parte (Sánchez N., 2007) indica que: "La puesta en práctica de estrategias gerenciales debe considerar como primer elemento a la cultura de la institución como agente no obstaculizador sino por el contrario, facilitador de los procesos, por eso debe ser punto de partida para cualquier diagnóstico"

En relación a lo anterior, (Rodríguez, 2011) describe: "La cultura organizacional como una nueva óptica institucional lo cual le permite a la gerencia comprender y mejorar las organizaciones y las 
instituciones para lograr tales fines”. El autor comenta que es necesario que la organización exija los siguientes aspectos:

- Un trabajador con el conocimiento para desarrollar y alcanzar los objetivos de la institución.

- Un proceso flexible ante los cambios introducidos por la institución.

- Una estructura plana, ágil, reducida a la mínima expresión de los objetivos organizacionales.

- Un sistema de trabajo participativo en las acciones de la institución.

Con estos argumentos la cultura organizacional, es la médula de una institución y por lo tanto está presente en todas las funciones y acciones que realizan sus miembros, además puede manifestarse a través de conductas significativas de los miembros de las organizaciones, las cuales facilitan el comportamiento en la misma. Así mismo, sin duda alguna es considerada como un hecho social que engloba diversidades y las unifica, liga materialidad e inmaterialidad.

\section{Metas y objetivos del comportamiento organizacional}

El autor (Amorós, 2007) en su libro acerca del Comportamiento Organizacional en busca del Desarrollo de Ventajas Competitivas, expresa los objetivos y metas de un CO los cuales se presentan a continuación:

- Describir: Sistemáticamente cómo se comportan las personas en condiciones distintas.

- Comprender: Por qué las personas se comportan como lo hacen.

- Predecir: El comportamiento futuro de los empleados.

- Controlar: Al menos parcialmente las actividades humanas en el trabajo. 
Con el primer objetivo describir al lograrlo permite que los administradores se comuniquen con un lenguaje común respecto del comportamiento humano en el trabajo. El segundo objetivo comprender: entienden las razones del porqué de su comportamiento y pueden entre otros lograr explicaciones, mejorar métodos, con el tercer objetivo predecir, es consecuencia del primero y el segundo, ya que al describir y comprender los gerentes, directivos, administradores, conocerán al personal, sus habilidades, relaciones intergrupales, tendrían la capacidad de predecir cuáles empleados son dedicados y productivos, y cuáles se caracterizarán por ausentismo, retardos u otra conducta perturbadora en determinado momento (de modo que sea posible emprender acciones preventivas). El último objetivo del comportamiento organizacional es controlar, los supervisores, gerentes, administradores, por ser responsables de los resultados de rendimiento, les interesa de manera vital tener efectos en el comportamiento, el desarrollo de habilidades, el trabajo de equipo, coordinación de esfuerzos y la productividad de los empleados.

Se puede decir que toda organización es una unidad social clasificada de forma consciente, conformado por personas, y que marcha con una base de relativa continuidad para llegar a sus metas delineadas, tomando en cuenta que exista una organización no basta con el conjunto de personas; ni siquiera es suficiente que todas ellas posean un propósito en común, se debe tomar en cuenta realmente y decisivo es que dichas personas se organicen o coordinen sus actividades, concretando la acción conjunta hacia el logro de unos resultados que, aunque sea por razones diversas, estimen todas ellas que les interesa conseguir, en las organizaciones desarrollan su trabajo los llamados gerentes, que son los individuos que supervisan las actividades y logran determinadas metas por medio de las demás personas, a las que se les llama subordinados.

\section{Características del comportamiento organizacional}


El autor (Garzón, 2010) comenta que: El comportamiento organizacional tiene una serie de características lo que concuerda con la opinión del autor (Ronnins\&Jude, 2009) de acuerdo a la opinión de ambos a continuación se expresan las caracteristicas:

- Identidad de sus miembros. Es la capacidad que tienen todos los trabajadores de sentirse identificados con la organización.

- Énfasis en el grupo. Las actividades del trabajo se organizan en relación a grupos y no a personas.

- Enfoque hacia las personas. Esto quiere decir que antes de tomar una decisión a nivel administrativo, se considera las repercusiones que podrían traer los resultados a cada uno de los miembros de la organización.

- La integración de unidades. Para trabajar de manera coordinada e independiente.

- Orientación a los resultados, lo cual se traduce en la organización centrada en los resultados o eventos, en vez de las técnicas y procesos usados para lograrlos.

- El control. Se establece el uso de reglas, procesos y supervisión para el control de la onducta de los individuos.

- Innovación y aceptación al riesgo. Se refiere a que los empleados sean innovadores arriesgados y agresivos.

- Los criterios para recompensar; es decir, cómo es que se distribuyen las recompensas, ya sean con aumentos de sueldos y ascenso de acuerdo con el rendimiento del empleado.

- El perfil hacia los fines, los medios.

- Atención al detalle. Se refiere al grado en que se espera que los empleados muestren precisión, análisis y atención por los detalles. 
- El enfoque hacia un sistema abierto, para que la organización controle esos cambios extremos.

Se puede especificar que cada una de estas características antes mencionadas son una continuidad que va de lo bajo hacia lo alto, en este sentido, se proporciona una imagen de los componentes del comportamiento que permite tener una orientación para realizar la investigación presente, cabe indicar, que las características se pueden combinar para comprender lo que determina el comportamiento organizacional así como su estructura.

\section{Elementos claves en el comportamiento organizacional}

Los autores (Soria \& López, 2008) en su libro sobre organizaciones y políticas públicas marcan que:

La conducta de los hombres en gran medida se basa en sus propias experiencias, por las normas que la sociedad construye para permitir una convivencia adecuada y equitativa entre sus integrantes, sean estas impuestas por el estado a través de las leyes y las sanciones correspondientes o por normas de carácter ético y/o moral, que le permite al hombre desarrollar sus potencialidades en la conducta individual o colectiva, dentro de la institución, se puede diferenciar dos formas de pensamiento opuestas las cuales quedan enmarcadas en los valores propios de los individuos, en la que el hombre busca su plena realización a través de su propio esfuerzo y con otros miembros de la sociedad y otra materialista en el que sobresalen sentimientos de individualidad, egoísmo, y competencia.

Cabe recalcar que el aumento de instituciones y por consiguiente el aumento en la incorporación de los individuos en ellas, estimula la transformación de las fuentes de valores, implicando con ello un 
menor impacto en la formación del individuo, de las consideradas como principales (familia y escuela, por ejemplo), y por consiguiente un cambio en su comportamiento.

\section{Variables del Comportamiento Organizacional}

Para el estudio del CO, la literatura especializada y algunos autores entre ellos (Chiavenato, 2009) y (Robbins, 2004), coinciden en recomendar el siguiente modelo básico, que atiende a tres niveles de análisis: individual, el cual consiste en la comprensión y dirección del comportamiento individual, de grupo atiende a la comprensión y dirección de grupos y procesos sociales y el organizacional radica en la comprensión y dirección de procesos y problemas organizativos.

\section{Figura 1. Niveles del Comportamiento Organizacional}

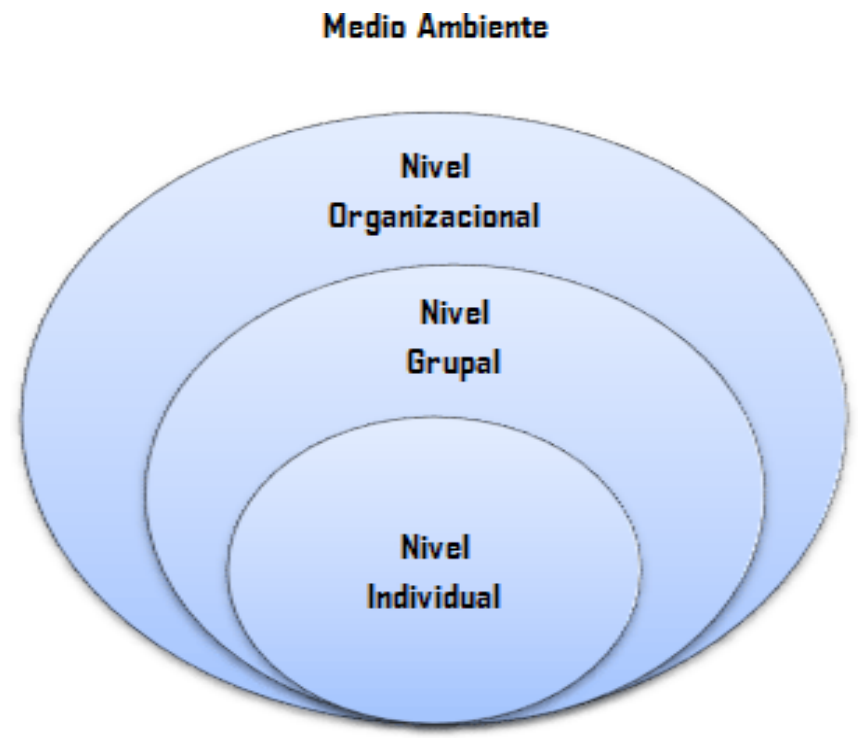

Fuente: (Chiavenato, 2009).

En la figura se observa que estos tres niveles de análisis que van desde una perspectiva micro a una perspectiva macro, bajo la consideración de la organización como sistema abierto, esta adherido a un medio externo llevado al conocimiento de cómo actúan para entender el comportamiento de la 
organización. Al pasar del nivel individual al nivel grupal en los sistemas de organización, se observa sistemáticamente el comportamiento en las organizaciones. Los tres niveles básicos son análogos a los bloques de construcción; cada uno se construye sobre el anterior y cada nivel es una consecuencia del anterior. Primeramente los individuos con sus características propias, luego el grupo y al final llegaremos al nivel del sistema organizacional donde se unirán los niveles anteriores como un sistema único, (Robbins, 2004).

Estos tres niveles se consideran una herramienta sumamente útil en los estudios de comportamiento organizacional, la gran claridad de dicho modelo radica, en que en la medida que se logra interrelacionar el conjunto de las variables en cada uno de los niveles se tiene certeza de la manifestación de estas en los resultados humanos, que es lo que busca conseguir toda organización sea esta pública o privada.

\section{El comportamiento de un Grupo}

El autor (Ceja, 2001) expresa que:

Todo grupo humano se concibe como un sistema social, constituido por las actividades, interacciones y los sentimientos que se dan entre los miembros del grupo. Los sistemas sociales poseen dos componentes, El sistema externo, constituido por las actividades, las interacciones y los sentimientos requeridos, así como los sentimientos dados, y por la relación existente entre estos elementos. El sistema interno, constituido por las actividades, las interacciones y los sentimientos que emergen espontáneamente entre los miembros del grupo a partir de lo que es requerido y de lo que es dado. 
En efecto, estos sistemas explican la relación entre el sistema externo, que es lo que establece y pone la dirección y el sistema interno que es lo que emerge espontáneamente, todo esto con la finalidad de explicar los procesos sociales más cercanos a las organizaciones, este comportamiento se ubica en el campo general de la conducta es decir, los actos de las personas, tratándose concretamente en las acciones de las personas en su trabajo.

Los autores (Davis \&Newstrom, 2002) opinan que:

Los grupos para atender sus objetivos, realizan entre sus miembros una cierta división del trabajo, cada miembro tendrá sus funciones. En todo grupo se desarrolla una estructura jerárquica informal y cada miembro tendrá su estatus diferente. El prestigio de un individuo en un grupo, puede depender de la aptitud por hacer prevalecer, en el comportamiento del conjunto de los miembros, los valores más fundamentales del grupo, o simplemente, por un criterio de antigüedad en la defensa de esos valores.

Sin embargo, (De Faria, 2005) dice que: "El grupo ejerce sobre los individuos una presión para que cada individuo tenga un comportamiento conforme a las normas. Esta coherencia, se realiza por parte del individuo al asociarse con gente con la que le une algún tipo de comportamiento"

La interdependencia de estas personas y la toma de conciencia de cada uno en la participación común, es lo que constituye un grupo, para poder cooperar, es necesario que los miembros del grupo compartan sus creencias, sus valores y las normas que regulan su relación, tomando en consideración que cada individuo asume un rol dentro del grupo, entendiendo como rol al tipo de comportamiento que determina el lugar del individuo en el grupo y los roles que deben cumplir es el método que permite estudiar actitudes y sentimientos de cada individuo. 
Relaciones de reciprocidad en las Organizaciones

Entre los grupos de interés de la organización se encuentran: directores, gerentes, empleados, obreros, sindicatos, entre otros y la organización existe fuerte relación de reciprocidad, es decir, la organización espera que los grupos de interés hagan contribuciones y les otorga estímulos, recompensas para que cooperen más, por otra parte los grupos de interés hacen aportaciones y a cambio de ello esperan obtener incentivos y estímulos.

"Cada una de las partes toma decisiones sobre continuar con sus inversiones. Este proceso es importante para comprender los intercambios que ocurren dentro y fuera de la organización" (Chiavenato, 2009). Estas relaciones de intercambio se visualizan en la siguiente figura.

Figura 2. Relaciones de Reciprocidad entre las personas y la Organización

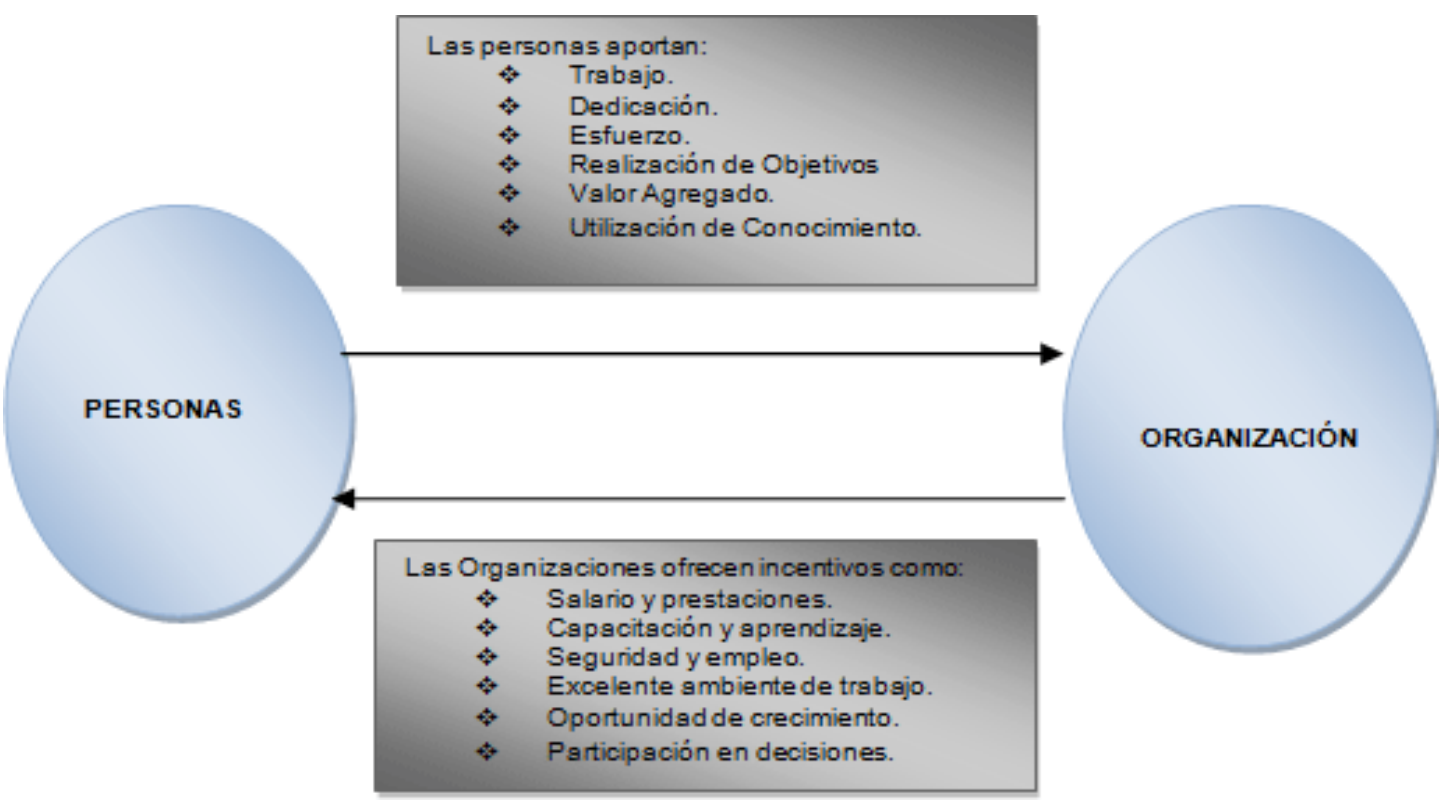

Fuente: (Chiavenato, 2009). 
En base de estos procesos, que sostienen la personalidad individual es posible identificar claramente un valor adaptativo, que se formula en las modalidades de mantención de la coherencia interna y de la igualdad en el transcurso de la vida, de manera de asegurar un sentido de diversidad subjetiva y de persistencia en el tiempo.

\section{Auditoría Pública}

El autor (Krohling, 2006) dice que:

El origen de la Auditoria, empieza como una necesidad social, proveniente del desarrollo de la economía, cuando la civilización occidental logro su paso de la Edad Media al periodo del Renacimiento, no se veían grandes transacciones de dinero, pero se dio inicio entre los reinos al préstamo de dinero, el cual tomo gran importancia, creando así, la necesidad de poder contar con una persona que fuera imparcial y pudiera dar fe de la transacción.

Sin embargo, (Mendez, 2008) opina que:

Es una actividad independiente, de apoyo a la función directiva, enfocada al examen objetivo, sistemático y evaluatorio de las operaciones financieras y administrativas realizadas; a los sistemas y procedimientos implantados; a la estructura orgánica en operación; y a los objetivos, planes, programas y metas alcanzados por las dependencias y entidades de la administración pública, con el propósito de determinar el grado de economía, eficacia, eficiencia, efectividad, imparcialidad, honestidad y apego a la normatividad con que se han administrado los recursos públicos que les fueron suministrados, así como la calidad y calidez con que prestan sus servicios a la ciudadanía. 
Mientras que (Estupiñan, 2006) menciona:

Un examen que pretende servir de base para expresar una opinión sobre la razonabilidad, consistencia y apego a los principios de contabilidad generalmente aceptados, de estados financieros preparados por una empresa o por otra entidad para su presentación al Publico o a otras partes interesadas.

“El examen crítico y sistemático de la actuación y los documentos financieros y jurídicos en que se refleja, con la finalidad de averiguar la exactitud, integridad y autenticidad de los mismo”. (Mendez, 2008)

Lo anteriormente descrito, demuestra que la auditoria empezó como aquella necesidad de control para evitar errores, desfalcos o previniendo que algunas personas se apropiaran de riquezas que no les pertenecían, debido a la aceptación las empresas públicas y privadas, han hecho de esta práctica, una herramienta necesaria, para el cumplimiento de sus objetivos, que les brinda la oportunidad de conocer en tiempo real su situación actual, para poder reaccionar oportunamente al entorno del mercado. Estas ilustraciones demuestran que la Auditoria pública es un proceso que tiene establecido una serie de parámetros, que hacen que se convierta en una herramienta esencial para las organizaciones, que les permite mediante la recolección de evidencias, determinar la confiabilidad y calidad de la ejecución de actividades realizadas por su organización, en congruencia a sus criterios, requisitos, políticas y procedimientos establecidos, para la toma de decisiones.

Normas generales de auditoría pública:

Normas Personales 
Primera.- Independencia: En la planeación, ejecución, elaboración de informes de auditoría y demás asuntos relacionados con su actividad profesional, el auditor público debe mantener soberanía de juicio, ser autónomo y objetivo, para proceder con independencia, ser independiente desde el punto de vista organizacional y mantener una actitud independiente.

Segunda.- Conocimiento técnico y capacidad profesional: El personal encargado de realizar la auditoría pública debe poseer en conjunto el conocimiento técnico y capacidad profesional necesarios.

Tercera.- Cuidado y diligencia profesionales: El debido cuidado y diligencia profesional en la realización de la auditoría y en la preparación del informe relativo, comprometen al auditor público a acatar las reglas de ética profesional y al cumplimiento de las normas generales de auditoría pública (Estupiñan, 2006).

\section{Normas sobre la Ejecución del Trabajo}

Cuarta.- Planeación: Previamente a la ejecución del trabajo de auditoría, el auditor deberá realizar una investigación para definir sus objetivos, alcances, procedimientos, recursos, tiempos y oportunidades de los mismos y precisarlo en los correspondientes programas.

Quinta.- Sistemas de Control Interno: El auditor público deberá efectuar un adecuado examen del control interno operativo y contable establecido.

Sexta.- Supervisión Del Trabajo De Auditoría: El personal debe ser cuidadosamente supervisado en la planeación y ejecución de la auditoría, en la preparación del informe y en el seguimiento de observaciones. 
Séptima.- Evidencia: El auditor público deberá realizar, con la amplitud que estime necesaria, las pruebas adecuadas para obtener evidencia de calidad que fundamente objetiva y razonablemente, sus conclusiones y recomendaciones.

Octava.- Papeles de Trabajo: Los procedimientos aplicados, las pruebas realizadas y la evidencia se deberán documentar en papeles de trabajo para soportar la opinión teniendo presente la naturaleza y características de los mismos estos son propiedad de la instancia fiscalizadora.

Novena.- Tratamiento de Irregularidades: El auditor deberá prestar especial atención a aquellas transacciones o situaciones que denoten indicios de irregularidades, haciéndola del conocimiento de las autoridades competentes (Estupiñan, 2006).

Las normas de la auditoría pública son consideradas una herramienta fundamental del Sistema Integral de Control y Evaluación de la Gestión Pública, ya que permite conocer la forma de que las dependencias y entidades de la Administración Pública realizan su gestión, establecen los requisitos mínimos que deberá observar el auditor público en el desahogo de sus programas de auditoría, con el propósito de contribuir al proceso de cambios estructurales que vive y demanda el país vigilando el uso eficiente de los recursos públicos y el cumplimiento oportuno, transparente y veraz del rendimiento de cuentas a la ciudadanía.

\section{Clasificación y tipos de auditoría pública:}

“Al analizar el amplio campo de aplicabilidad de la Auditoria pública y teniendo en cuenta su evolución en el tiempo, se ha encontrado como necesidad la especialización de su actividad, de acuerdo al objeto, destino, técnicas, métodos, etc., brindando una herramienta que de acuerdo a su campo de aplicación que permita en una forma eficaz el logro de los objetivos planteados" (Alvin, 
2000). Por lo anterior se ha clasificado por la procedencia del auditor y por su área de aplicación, así:

\section{Auditoría interna:}

Es desarrollada por personal que depende de la empresa, se realiza al servicio de la alta dirección, como mecanismo de control que brinda un dictamen interno sobre las actividades de toda la empresa, que permite ver que las políticas y procedimientos establecidos al interior sean aplicados de forma correcta a todas las áreas.

(Mendez, 2008) afirma que: "El órgano asesor de la dirección que busca la manera de dotar a la empresa de una mayor eficiencia mediante el constante y progresivo perfeccionamiento de políticas, sistemas, métodos y procedimientos de la empresa".

Dentro de las actividades que se deben desarrollar se encuentra: Identificación de los riesgos, Evaluar susceptibilidades a distorsiones, diseñar procedimientos de auditoria que permita evaluar el diseño la implementación y efectividad. Una ventaja importante de la Auditoria Interna, es la habilidad que tiene para minimizar los riesgos, reducir costos, eliminar complejidad de los controles con el fin de mejorar la competitividad y proteger el valor de la compañía.

\section{Auditoria externas:}

“Es efectuada por personal externo a la empresa en donde examina y evalúan sus operaciones, para emitir una opinión veraz y técnica del sistema de control que se está desarrollando en esa área”.

(Thevenet, 2010), califican la Auditoria como externa por su condición de legalidad y porque el auditor es ajeno a la empresa. 
Hay que tomar en consideración que al no tener ninguna dependencia con la empresa, el trabajo del auditor es totalmente independiente y libre de cualquier influencia por parte de las autoridades de las empresas, generando así una mayor confiabilidad a terceros sobre los resultados arrojados.

\section{Importancia de la auditoria pública:}

El autor (Fundación EcaGlobal, 2006) en su estudio expone:

Las auditorías surgieron durante la revolución industrial, como una medida orientada a identificar el fraude, con el tiempo esta visión cambió, y hoy es una herramienta muy utilizada para controlar el alineamiento de la organización con la estrategia propuesta por la administración y asegurar un adecuado funcionamiento del área financiera. Actualmente se han multiplicado los tipos de auditorías, respondiendo a una creciente complejidad empresarial.

En su obra (Sánchez J. 2001) también expresa:

La mayoría de empresas pequeñas no tienen la obligación de hacer auditorías y no le dan importancia, pero una auditoría puede ayudar a las organizaciones a optimizar sus operaciones y lograr importantes ahorros de costos. Una auditoría interna puede ayudar a los gerentes a establecer medidas para lograr un buen control financiero y de gestión, al realizar un análisis del balance se pueden identificar con claridad aquellas situaciones que constituyen un riesgo y lo que podría ser una oportunidad financiera; además, al realizar un análisis de la cuenta de resultados se puede planificar algunas estrategias para lograr mayores niveles de ahorro y rentabilidad. 
Mientras que (Bustamante, 2008) opina que:

Se debe realizar una auditoría al menos una vez al año, de esa forma se logrará un mejor control sobre los procesos de gestión en una empresa, permitiendo un crecimiento ordenado que garantice la sostenibilidad de la organización a largo plazo. Se pueden realizar auditorías internas, contando con el personal de la organización para realizar un control de los procesos administrativos y analizar los estados financieros de la organización, pero además se puede contratar a empresas externas para que realicen una auditoría objetiva que permita identificar en la organización los principales riesgos que deberían resolverse y afinar las estrategias para lograr que se ajusten a lo que espera la gerencia.

Mediante a las auditorías se puede identificar los errores cometidos en las organizaciones y se puede enmendar a tiempo cualquier falla en la ejecución de la estrategia, para tomar medidas que permitan retomar el rumbo correcto en la empresa, es preciso realizar una auditoría cuando existen evidencias de gastos excesivos, incumplimiento de proyectos, quejas de los clientes, alta rotación de recursos humanos y cuando los resultados se alejan de los que la empresa espera.

Importancia del comportamiento organizacional en la auditoría pública:

Con el objeto de equilibrar el examen integral de las empresas, es necesaria la aplicación de la técnica de la auditoria en el área de la administración.

Esto se debe a que generalmente es el área financiera a la que se le da atención, quedando un desequilibrio en el examen que se realiza en la empresa. En alguno de los casos en este campo se aplica la auditoria llevando implícito el carácter administrativo, donde el auditor moderno ha querido separarlos, dándole así a la auditoria administrativa su propia esencia y 
naturaleza de la actividad que se realiza en las empresas, y así completar el examen integral en cada área funcional (Wittington\&Pany, 2005).

La administración pública en los últimos tiempos ha crecido, y han aparecido nuevos retos y nuevas formas en su aplicación así lo afirma (Estupiñan, 2006) donde: “El aparecimiento tecnológico que le exige nuevas respuestas a las interrogantes que antes contestaba fácilmente, la administración ha crecido y cada día se hace más complicado su manejo por lo que es necesario, darle importancia a los aspectos más específicos para su mejor desarrollo" La circunstancia anterior permite una observación cuidadosa, un estudio analítico que esté al alcance de los que dirigen las empresas.

Anteriormente el examen de la empresa se limitaba principalmente a la situación financiera, al manejo de las finanzas al manejo de los números, es decir, cuantitativamente

Lo que significaba una investigación minuciosa para averiguar la situación financiera y descubrir fraudes y errores, sin embargo hace falta la revisión, el estudio y la evolución de la empresa en su conjunto, en su composición y estructura, organizada en cada una de las funciones que la integran desde la cúpula hasta el último nivel de la organización (Chiavenato, 2009).

"La auditoría como disciplina es importante tanto en la empresa pública como en la empresa privada, así en la empresa agrícola, industrial y comercial, en la empresa pequeña, mediana y grande, en cualquier unidad organizativa de la empresa de la empresa, gerencia, división, departamento, sección, unidad”. (Fundación Eca Global, 2006). De acuerdo a esto el autor (Wittington\&Pany, 2005) dice que: “La auditoría pública se ha considerado como el examen completo y constructivo de la estructura organizativa de una empresa, institución o departamento 
gubernamental o de cualquier otra entidad, así como de sus métodos de control, medios de operación y empleo que dé a sus recursos humanos y materiales”.

El autor (Arens\&Loebbecke, 2000) en su estudio expone:

La auditoría se genera por múltiples causas dentro de la administración de una empresa; su género, su especie pertenece al área del control, que ubicado mediante evaluaciones y exámenes que se realizan en los diferentes elementos mantienen el ajuste y el orden dentro del que hacer de la administración general. A continuación se presenta una lista de algunas de las causas ordinarias que pueden contribuir a una deficiente administración o a una empresa mal llevada, estas son:

- Inutilidad de los objetivos por ser estos ambiguos y confusos.

- Violación de autoridad - responsabilidad que ha sido asignada.

- Falta de iniciativa por falta de delegación de autoridad.

- Interferencia entre línea y asesoría, definición de funciones.

- Organización costosa innecesaria.

- Difícil y costoso control.

- Demasiados o pocos jefes, área de control adecuado a las necesidades.

- Deformación de la comunicación y de la información entre los niveles de la organización.

- Conflictos de competencia y personal falto de motivación.

- Difícil visión integral de conjunto de la misión de la empresa.

- Decisiones lentas, demoras a la orden del día.

- Duplicidad de funciones, traslape de actividades que generan confusión. 
- Gerente autoritario, no existe comunicación y participación.

- Falta de coordinación, privan los intereses personales a los generales.

- Falta de trabajo creativo, se deja por fuera el cambio por medio de la imaginación.

- Despilfarros, desperdicio de producto, de tiempo y dinero.

- Mucha ostentación, sin darle el verdadero valor al uso de los materiales.

- Falta de financiamiento oportuno. etc.

Lo anterior se evita en lo posible con exámenes periódicos que se hagan en el informe de auditoría pública, la importancia de estos exámenes es que se realizan en el transcurso del tiempo, proporcionan confianza de la buena marcha de cualquier negocio, esto no solo es evidente, en países desarrollados sino que también en los países en vías de desarrollo, donde cada día se puede sugerir la necesidad instauración de unidades especializadas de este tipo en las empresas que no contaban hasta el momento con ellas.

La generalización de la labor de auditoria es con el objeto de amarrar y de maximizar la eficacia del control interno de la empresa

Es en este proceso donde la técnica de auditoria administrativa aplicada en empresas de cierta magnitud se constituye un auxiliar fundamental de la dirección empresarial, ya que la constitución de los objetivos implica la existencia de un sistema de control dirigido a corregir las desviaciones e identificar causas, con relación a las metas preestablecidas (Burbano, 2001).

Los que significa que hay que llevar a cabo un análisis exhaustivo de la organización por el auditor público o el que haga las veces de él; por lo que debe poner en juego toda su imaginación para hacer 
realidad el axioma "Siempre existe una forma mejor de realizar las cosas" y que generalmente, puede conseguirse por medio de la investigación que se realice, tomando como base los recursos de que dispone el organismo social. (Burbano, 2001) afirma que: "Los factores externos, o sea, el entorno de la unidad empresarial tiene una atención especial pues su influencia es determinante para la operación de la empresa por lo tanto su examen debe comprender desde el Medio Ambiente que debe cubrir Localización - fuerza de trabajo - aspectos legales - cargas fiscales - servicios mercados - materia prima-comunicaciones, etc".

Las áreas de investigación pueden incluir una apreciación de los métodos y procedimientos, planeación de entregas, medidas de actuación, costos de ingeniería de proyectos, estimación y fijación de precios, comunicaciones, equipos electrónicos de procesos de datos, efectividad de los directivos involucrados. Como ya se había especificado, todo lo anterior implica prácticamente cubrir en forma integral el área de control interno administrativo u operativo, o sea el campo de la función control de la dirección superior.

\section{Auditoría a la Evaluación del Desempeño}

Debido a la importancia del proceso de evaluación del desempeño para las organizaciones, muchas de éstas están invirtiendo recursos para tener la opinión desde una visión de un experto respecto a la razonabilidad del proceso, acudiendo a los auditores de recursos humanos.

La auditoría de recursos público es compleja, puesto que: es un procedimiento que tiene por objeto revisar y comprobar, bajo un método especial, todas las funciones y actividades que en materia de personal se realizan en los distintos departamentos, para determinar si se ajustan a los programas establecidos y evaluar si se cumplen los objetivos y políticas fijadas 
en la materia, sugiriendo en su caso, los cambios y mejoras que deban hacerse para el mejor cumplimiento de los fines de la administración de personal (Reyes A. , 2006).

Sin embargo el autor (Burbano, 2001) opina que a pesar de su complejidad, posee múltiples beneficios, entre los cuales menciona los siguientes:

- Crea una imagen de reconocimiento al recurso humano de la firma ante directivos y subalternos.

- Incentiva la responsabilidad del personal de la empresa.

- Clarifica los derechos, deberes y responsabilidades de cada individuo.

- Permite identificar problemas críticos del personal antes de repercutir en el ambiente organizacional.

- Economiza costos al aplicar procedimientos eficaces de administración de personal.

- Permite la medición de eficiencia de los recursos humanos frente a los recursos materiales.

- Asegura un mejor cumplimiento de los requerimientos legales y sociales.

- Por último, el cálculo del costo de vinculación y desarrollo del personal, así como la determinación de su valor, es importante en la fijación de estrategias y políticas de personal.

Muchos autores se han referido a lo que significa la auditoría pública y la importancia que ésta tiene para las organizaciones, sin embargo, actualmente está identificándose una nueva tendencia en la que ciertos autores se están refiriendo más que a la auditoría genérica de recursos humanos, a la auditoría específica que se realiza a cada una de las áreas; por ejemplo, auditoría a la capacitación, al reclutamiento, al proceso de selección, entre otros. 
En esta definición se encuentra incluida también la auditoría de evaluación de desempeño. Si bien actualmente, no son muchos los autores ni las publicaciones que han abordado este tema, encontramos algunos que hacen alusión al concepto y mencionan ciertos aspectos que se deben evaluar en una auditoría a las diferentes funciones de los recursos humanos, incluyendo también los principales aspectos que se deben evaluar de la función de evaluación del desempeño. (Estupiñan, 2006)

Para finiquitar, es importante recalcar que para poder realizar una auditoría de calidad a la evaluación de desempeño es básico y fundamental, para un auditor, que conozca claramente las etapas del proceso y todos sus componentes; es vital, además, que conozca una definición del concepto, tener noción de lo que significa la evaluación del desempeño y conocer y considerar todas las variables exógenas que pueden afectar su auditoría pública, especialmente la de evaluación de desempeño.

\section{La crítica de la determinación de lo público por lo privado}

El autor (Estupiñan, 2006) considera que:

La tarea de la auditoría pública contemporánea consiste precisamente en ponerla en primer plano, como exigencia primaria y fundamental, si se admite que, a través de la auditoría pública, la realidad pública puede manifestarnos sus determinaciones, límites, propiedades y categorías, debemos admitir que no es tal de volver imposible a la auditoría pública. Su tarea no queda fuera de la realidad pública, sino que pasa en alguna forma a constituirla, y a construirla. Se puede considerar también que el ejercicio y práctica de la auditoría pública depende de la realidad pública hacia la cual esta se dirige, y es evidente que existe una 
relación entre la auditoría pública y su realidad, que debe ponerse en claro, pero esta relación no está determinada y se debe determinar, y es por tanto un problema.

La situación pública se manifiesta en la crisis actual, pero en el ámbito profesional de la auditoría pública se ha reducido, como se señala

Como conclusión destacamos que la normativa contable y auditora no ha sido uno de los elementos principales detonantes de la actual crisis, aunque un analista económico asevere Lo que experimentamos no es una crisis del capitalismo, es una crisis de finanzas, democracia, globalización $\mathrm{y}$, a fin de cuentas, de ética", y un filósofo afirme sobre el surgimiento de la crisis, que desde la década de los años sesenta, asistimos a una transformación que paulatinamente ha desembocado en la gran crisis no se trata, como se afirma en los medios de comunicación, de una mera crisis política, o de una crisis social, o económica, no. La crisis real es una crisis histórica y es de tal envergadura que nos impide ver sus resultados claramente. Es una crisis verdadera, de allí su oscuridad, cabe destacar que ésta afirmación fue realizada en el año 2005, y su importancia radica en no reducir el problema de la crisis a una fecha, a un año, o una cuestión pasajera y coyuntural (Burbano, 2001)

Como lo expresa el autor (Arens \& Loebbecke, 2000):

Lo público es aquello que es de interés general, debe estar a la luz, todos deben poderlo ver. No solamente la auditoría debe comportar elementos claros y sencillos, la contabilidad pública también, redefinirlas es una exigencia, si la auditoría pública aspira a garantizar al ciudadano la posibilidad de evaluar el manejo de los fondos públicos de manera responsable y con probidad, no podrá prometer soluciones definitivas, ni seguridades y garantías 
infalibles, como las disposiciones y lineamientos aprobados por los gobiernos e instituciones que continúan prometiendo lo que no se puede cumplir.

Los resultados de escribir o reflexionar sobre la auditoría, se encuentra en la palabra misma de auditoría, que aparece estar autorizada para otorgarse su propia validación o criterio de verdad, así el término de auditoría se presenta ineludible e indisolublemente en cualquier actividad humana donde se ejerce la revisión, como en los diferentes tipos de auditorías existentes, pero no existe una uniformidad (claramente no se trata de confundir la normatividad que ha regido el pensamiento de la auditoría que "contra viento y marea" continua sosteniendo, en lo esencial, la misma direccionalidad, con la libertad individual o grupal de auditores que promueven una concepción particular de auditoría o que deciden si adoptan las normas internacionales de auditoría, pero que se ha carecido de la reflexión o fundamentación de una Teoría de la Auditoría, que permita re-construir la divisoria de lo público y lo privado para observar de nuevo la realidad) al respecto, ni mucho menos existe una historia de la auditoría que dé cuenta de cada una de ellas. Porque las auditorías surgen y desaparecen, es decir, tienen un principio y un final.

\section{Conclusiones.}

Debido a todos los problemas administrativos que sé han venido presentado con el avance del tiempo, se han creado, nuevas dimensiones en el pensamiento administrativo, considerando una de estas la auditoria pública, la cual es un examen detallado de la administración de un organismo social, realizado por un profesional al que se le llama auditor, que se define como una nueva herramienta de control y evaluación considerada como un servicio profesional para examinar integralmente un organismo social con el propósito de descubrir oportunidades para mejorar desde un mejor comportamiento organizacional hasta toda la parte administrativa, tomando en cuenta 
todas las investigaciones realizadas para dicho trabajo, siendo una actividad dinámica, aplicada formalmente en toda empresa u organismo público, independientemente de su magnitud y objetivos con el fin de lograr eficiencia.

Durante el desarrollo de la investigación se observó que la auditoría pública es una necesidad en la actividad empresarial en estos tiempos, por lo tanto en la especialidad de campo administrativo se vuelve imperiosa su aplicación para el logro de una efectiva administración, siendo una ventaja competitiva que le permite a la administración ser eficaz y lograr niveles de eficiencia, pilares que le permiten a cualquier empresa competir en el mercado, y visualizar de inmediato la posibilidad de ubicar a la empresa en la ruta correcta de su desarrollo administrativo y operativo para el logro de los objetivos. Por esta razón, se considera una herramienta preventiva de los efectos negativos que ocasiona la mala coordinación, relación y la valuación de cada uno de los elementos que forman la gestión gerencial, comparada como un antivirus administrativo que permite anticiparse ante las amenazas que se presentan.

Además de la auditoria pública se observó, que el comportamiento organizacional es uno de los temas de mayor relevancia en una empresa y que de ésta depende la evaluación que se tenga al momento de la auditoria. Está enfocado en la necesidad de una nueva perspectiva de negocio llevado a un sistema o conjunto y a un grupo de personas que se interrelacionan y analizan problemáticas para conseguir un fin determinado bajo esta perspectiva, para resaltar algunos aspectos de análisis que lo conforman y lo vuelven una praxis indispensable con el fin de mejorar la relación entre los colaboradores de una organización, como eje dinamizador de la eficiencia y eficacia de las empresas. 
La clave de un exitoso proceso acertado en el desarrollo de las organizaciones, está en la actitud de las personas que participan en ella, de ahí que el comportamiento organizacional sea una herramienta necesaria para beneficio de todo tipo de empresas, cuyo resultado de una adecuada gestión organizacional obedecerá en gran medida a cómo se da la motivación, la creatividad, la productividad y el sentido de pertenencia de los miembros de la organización para lograr un mejor uso del capital humano, generando ventajas competitivas que contribuyan al aumento de los beneficios de la empresa,

Los funcionarios responsables de disponer de dichos fondos deben asegurarse de que sus decisiones son legales y éticas, así como que dichas decisiones resulten en servicios públicos eficientes, económicos y eficaces, donde las personas que tienen a su cargo las Entidades Públicas deben rendir cuentas sobre la manera en que manejaron los recursos confiados a ellos, este principio de rendición de cuentas se aplica en su totalidad al sector público, desde representantes electos que son quienes rinden a la sociedad, hasta los funcionarios públicos que son quienes rinden a los representantes electos. En todos los niveles existe la obligación de demostrar que se están siguiendo las prácticas administrativas adecuadas todo esto mediante una buena auditoría pública.

\section{Bibliografia.}

Alles, M. (2008). Comportamiento organizacional. . Buenos Aires: Granica S.A. ediciones.

Alvin, A. A. (2000). Auditoria Un enfoque Integral. Editorial Océano. Enciclopedia de la Auditoria. Amorós, E. (2007). Comportamiento Organizacional. En Busca del Desarrollo de Ventajas Competitivas. .s/n: EUMEDNET .

Arens, A., \&Loebbecke, J. (2000). Auditoría. Un enfoque integral (6a ed.). . México: Prentice Hall Hispanoamericana.

Burbano, J. (2001). Auditoría de personal (2 ${ }^{a}$ ed.). Colombia: ECOE Ediciones. 
Bustamante, K. (2008). Auditoría al proceso de evaluación del desempeño. Argentina: Macchi.

Ceja, G. (2001). Planeación y Organización de Empresas. . México: Décima Primera edición. Editorial Mc Graw Hill. INTERAMERICANA EDITORES, S.A. Méjico.

Chiavenato, I. (2009). Comportamiento Organizacional. La Dinámica del éxito en las organizaciones (Segunda Edición ed.). México: Editorial Mc Graw Hill. INTERAMERICANA EDITORES, S.A. Méjic.

Davis, K., \&Newstrom, J. (2002). Comportamiento humano en el trabajo . México: Editorial Mc Graw Hill. INTERAMERICANA EDITORES, S.A. Méjico.

De Faria, F. (2005). Desarrollo Organizacional. Enfoque Integral. México: Editorial Limusa. Grupo Noriega Editores. Méjico.

Estupiñan, R. (2006). Administracion del E.R.B y la Auditoria.s/n: Ed. Ecoe Ediciones.

Fundación EcaGlobal (2006). El auditor de calidad.Madrid : Fundación Confemetal.

Garzón, M. (2010). El Desarrollo Organizacional y el Cambio Planeado. Colombia: Centro Editorial Universidad del Rosario. Bogotá.

Gibson, J., Ivancevich, J., Donnelly, J., \&Konopaske, R. (2007). Organizaciones. Comportamiento, Estructura y Procesos. México: Editorial Mc Graw Hill. INTERAMERICANA EDITORES, S.A. Méjico.

Gil, I., Ruiz, L., \& Ruiz, J. (1997). La nueva dirección de personas en la empresa. Madrid: McGraw-Hill.

Hellriegel, D., \&Slocum, J. (2009). Comportamiento Organizacional.s/n: Cengage Learning Editores. Méjico.

Krohling, M. (2006). Auditoría de la Comunicación Organizacional, en: Rebeil Corella, M. A. (Coord.). Comunicación Estratégica en las Organizaciones. México: Trillas.

Mendez, H. (2008). Auditoria: Concepto, Clases y Evolucion. México: Ed. Mc Graw Hill.

Reyes, A. (2005). Administración de personal 1: Relaciones humanas. México: Limusa.

Reyes, A. (2006). Relaciones humanas. México: Limusa.

Robbins, S. (2004). Comportamiento Organizacional. Teoría y práctica (10a Edición. Tomo I ed.). Pearson Educación de Méjico. S. A. Prentice-Hall INC. Méjico.

Rodríguez, E. (2011). Gestión competitiva del gerente educativo para el cambio de la cultura organizacional dentro del marco de la educación robinsoniana. Carabobo: Universidad de Carabobo. 
Walter M. Mendoza-Zamora; Jessenia H. Morán-Chilán; Vanessa G. Fernández-Álava; Ana del Rocío Lino-Calle

Ronnins, S., \&Jude, T. (2009). Comportamiento organizacional. México. México: ediciones Pearson educación de México S.A. de c.v.

Sánchez, J. (2001). Análisis de los principales problemas que se presentan en la auditoría de procesos de capacitación. Contabilidad y Auditoría.. Madrid: McGraw Hill.

Sánchez, J., \& Bustamante, K. (2008). Auditoría al proceso de evaluación del desempeño. Contabilidad y Auditoría. México: Prentice Hall.

Sánchez, N. (2007). Propuesta de estrategias gerenciales para el cambio de la cultura organizacional en la Unidad Educativa Cabriales. Carabobo: Universidad de Carabobo.

Soria, R., \& López, C. (2008). Organizaciones y políticas públicas: una mirada desde el noroeste. México: ediciones Pearson educación de México S.A. de c.v.

Thevenet, M. (2010). Auditoria de la cultura empresarial. . Madrid: ediciones Díaz de santos.

Wittington, O., \&Pany, K. (2005). Principios de auditoría (14a ed.). México: McGraw-Hill.

Zapata, Á., Murillo, G., \& Martínez, J. (2007). Organización y Management; naturaleza, objeto, método investigación y enseñanza. Cali: Universidad del Valle. 